\title{
New prospects for continuous gravitational wave detection from binary systems in the advanced detector era
}

\author{
Paola Leaci* \\ Dip. di Fisica, Università di Roma "Sapienza" and INFN, P.le A. Moro, 2, I-00185 Rome, Italy \\ *E-mail: paola.leaci@roma1.infn.it
}

Reinhard Prix

Max-Planck-Institut für Gravitationsphysik, Albert-Einstein-Institut, D-30167 Hannover, Germany

\begin{abstract}
Gravitational wave detection is eagerly expected as one of the major discoveries of the next decade. A worldwide effort is currently underway, building ever-more-sensitive detectors and developing more powerful data-analysis tools. Continuous gravitational waves (CWs) are a promising class of signals for advanced detectors, and a particularly interesting type of CW sources are neutron stars in low-mass X-ray binaries, with Scorpius X-1 being the most remarkable representative. We describe the details of a project aimed to perform directed searches for CWs from binary systems. We use a search algorithm based on coherently computing a maximum likelihood statistic for a bank of signal templates, and then incoherently summing this statistic over the segments in which the entire data set has been previously split. The current strategy strongly relies on the derivation of precise analytic expressions for the (coherent and semicoherent) phase metrics of $\mathrm{CW}$ sources in low-eccentricity binary systems, for the two regimes of long and short segments compared to the orbital period. As an application of the metric template expressions, we estimate the optimal achievable sensitivity of an Einstein@Home directed search for Scorpius X-1, under the assumption of sufficiently small spin wandering, and using data from the upcoming advanced detectors. We find that such a search would be able to beat the torque-balance level up to a frequency of 500-600 $\mathrm{Hz}$, if orbital eccentricity is well-constrained, and up to a frequency of $\sim 160-200 \mathrm{~Hz}$ for more conservative assumptions about the uncertainty on orbital eccentricity.
\end{abstract}

\section{Introduction}

Continuous gravitational waves (CWs) are a promising class of signals for the advanced detectors currently under construction. ${ }^{1,2}$ These signals would be emitted from spinning neutron stars (NSs) with non-axisymmetric deformations. ${ }^{3}$ A particularly interesting type of searches for CW signals are those directed to Scorpius X-1. To this purpose, several new pipelines have been developed and compared. ${ }^{4}$

A coherent strategy for extracting faint CW signals buried in noisy data using standard matched-filtering techniques was derived in Ref. 5. The resulting detection statistic is the so-called $\mathcal{F}$-statistic. In order to increase sensitivity beyond existent methods, and to be able to effectively absorb large amounts of computing power (such as those provided by Einstein@Home ${ }^{6}$ ), it is necessary to consider general long-segment semicoherent methods, by stacking coherent $\mathcal{F}$-statistic segments ${ }^{7}$ and extending such methods to the search for CWs from binary systems.

Key ingredients required for building a semicoherent "StackSlide" search are the coherent and semicoherent parameter-space metrics. ${ }^{8,9}$ 


\section{Detection Statistic and Metric Mismatch}

As shown in Ref. 5, matched-filtering the data $x(t)$ against a template $h(t ; \mathcal{A}, \lambda)$, and analytically maximizing over the unknown amplitude parameters $\mathcal{A}^{\mathrm{a}}$, results in the coherent statistic $\widetilde{\mathcal{F}}(x ; \lambda)$. Using the $\mathcal{F}$-statistic means that we need to search explicitly only over the phase-evolution parameters $\lambda^{\mathrm{b}}$.

We focus on one particular semicoherent approach, referred to as StackSlide, which consists of dividing the total amount of data $T_{\text {obs }}$ into $N$ segments of duration $\Delta T$, such that $T_{\mathrm{obs}}=N \Delta T$ (in the ideal case of gapless data). The coherent statistic is then computed over all segments $\ell=1 \ldots N$, and combined incoherently by summing $\widehat{\mathcal{F}}(x ; \lambda) \equiv \sum_{\ell=1}^{N} \widetilde{\mathcal{F}}_{\ell}(x ; \lambda)$. This statistic follows a (non-central) $\chi^{2}$ distribution with $4 N$ degrees of freedom and a non-centrality parameter $\widehat{\rho}^{2}\left(\mathcal{A}, \lambda_{s} ; \lambda\right)$, which is given by $\widehat{\rho}^{2}=\sum_{\ell=1}^{N} \widetilde{\rho}_{\ell}^{2}$ in terms of the per-segment coherent SNRs $\widetilde{\rho}_{\ell}{ }^{c}$.

In order to systematically search a parameter space $\mathcal{P}$ using the $\mathcal{F}$-statistic, we need to select a finite sampling $\left\{\lambda_{t}\right\} \subset \mathcal{P}$ of the parameter space, commonly referred to as a template bank, and compute the statistic over this set of templates. A signal with parameters $\lambda_{s} \in \mathcal{P}$ will generally not fall on an exact template, and we therefore need to characterize the loss of detection statistic $\mathcal{F}$ as a function of the offset $\delta \lambda=\lambda-\lambda_{s}$ from a signal. This is generally quantified using the expected statistic $E[2 \mathcal{F}]$, namely as the relative loss in the non-centrality $\rho^{2}$ :

$$
\mu_{0} \equiv \frac{\rho^{2}\left(\mathcal{A}, \lambda_{s} ; \lambda_{s}\right)-\rho^{2}\left(\mathcal{A}, \lambda_{s} ; \lambda\right)}{\rho^{2}\left(\mathcal{A}, \lambda_{s} ; \lambda_{s}\right)},
$$

which defines the measured $\mathcal{F}$-statistic mismatch function. By Taylor-expanding this up to second order in small offsets $\delta \lambda$, and neglecting the dependence on the amplitude parameters $\mathcal{A}$, we obtain

$$
\mu_{0}\left(\mathcal{A}, \lambda_{s} ; \lambda\right) \approx \mu\left(\lambda_{s} ; \lambda\right) \equiv g_{i j}\left(\lambda_{s}\right) \delta \lambda^{i} \delta \lambda^{j},
$$

with $g_{i j}$ being the phase metric, expressible in terms of phase derivatives (see Ref. 9).

\section{Numerical Tests of the Metrics}

We present numerical tests performed on the parameter-space metrics derived in Ref. 9 assuming a low-eccentricity binary orbit and focusing on two limiting regimes, namely the long-segment limit (LS) where $\Delta T \gg P$, and the short-segment limit (SS) where $\Delta T \ll P$, with $P$ the source orbital period. These tests consist of comparing the predicted metric mismatches $\mu$ of Eq. (2) against measured $\mathcal{F}$-statistic

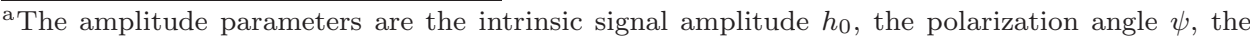
initial phase $\phi_{0}$, and $\cos \iota$, with $\iota$ being the inclination angle between the line of sight to the neutron star and its rotation axis.

${ }^{b}$ The phase parameters are the intrinsic signal frequency, the frequency time derivatives ("spindown" terms), the source sky position, and binary orbital parameters.

${ }^{\mathrm{c}}$ The expectations for $\widetilde{\mathcal{F}}$ and $\widehat{\mathcal{F}}$ are $E[2 \mathcal{F}(x ; \lambda)]=4+\widetilde{\rho}^{2}\left(\mathcal{A}, \lambda_{s} ; \lambda\right)$ and $E[2 \widehat{\mathcal{F}}(x ; \lambda)]=4 N+$ $\widehat{\rho}^{2}\left(\mathcal{A}, \lambda_{s} ; \lambda\right)$, respectively.
} 
mismatches $\mu_{0}$ of Eq. (1) obtained via signal software injections. The quantity used for this comparison is

$$
\varepsilon\left(\mu_{0}, \mu\right) \equiv \frac{\mu_{0}-\mu}{0.5\left(\mu_{0}+\mu\right)} \in[-2,2] .
$$

Phase and amplitude parameters are randomly drawn from uniform distributions according to what described in Ref. 9, and software injections and recovery are performed using the machinery detailed in Ref. 9. In the following we highlight the results obtained by computing the semicoherent parameter-space metric components for the LS regime. The scale for the orbital period used here is $P_{0}=2 \mathrm{~h}$, with segments of fixed length of $\Delta T=1 \mathrm{~d}$ and varying $T_{\text {obs. }}$. In total we used 10 values of $T_{\text {obs }}: 1 \mathrm{~d}, 10 \mathrm{~d}$, and $30 \mathrm{~d}$ up to $100 \mathrm{~d}$ in steps of $10 \mathrm{~d}$. The results of these tests are shown in Fig. 1 for the total of 20000 trials performed (2000 trials for each $T_{\text {obs }}$ value). We see that the agreement between measurements and predictions is
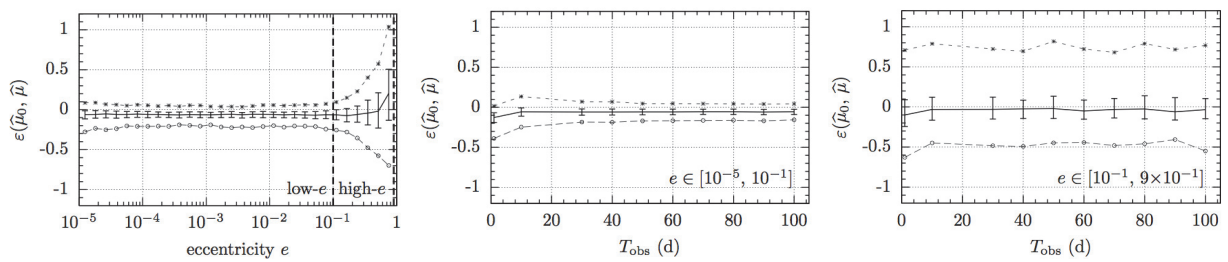

Fig. 1. Results for the semicoherent LS metric tests assuming an orbital period of $P_{0}=2 \mathrm{~h}$ and fixed segment length of $\Delta T=1 \mathrm{~d}$. Left panel: Relative error $\varepsilon\left(\widehat{\mu}_{0}, \widehat{\mu}\right)$ vs eccentricity $e$. The dashed vertical line denotes the boundary between a "low-e" range $e \in\left[10^{-5}, 0.1\right]$ and a "high- $e$ " range $e \in[0.1,0.9]$. Middle and Right panels: Relative error $\varepsilon$ vs observation time $T_{\text {obs }}$. The solid lines in all the panels denote the median value; the error bars correspond to the 25th-75th percentiles; the circles and the stars denote the 2.5 th and 97.5th percentiles, respectively.

generally good in the low-eccentricity regime $e<10^{-1}$ (left and middle plots), while rapidly degrading at higher eccentricites (right plot). The case $T_{\text {obs }}=\Delta T=1 \mathrm{~d}$ corresponds to the single-segment coherent case, and antenna-pattern effects are expected to play a role for observation times of order a day. By performing analogous Monte Carlo simulations for the coherent LS regime, and for both the coherent and semicoherent SS regime, we observe that the agreement between metric mismatch predictions and the measured relative loss in $\mathcal{F}$-statistic is generally very good (typically no worse than $\sim 10-30 \%$ ) within its range of applicability. The first-order small-eccentricity approximation starts to noticeably degrade only above $e \gtrsim 0.1$, except in the coherent SS regime where it breaks down above $e \gtrsim 5 \times 10^{-3}$.

\section{Sensitivity Estimates}

Applying the fast and accurate method developed in Ref. 10, and using the metric template counts computed in Sec. VA of Ref. 9, we semi-analytically estimate the optimal achievable StackSlide sensitivity of a directed search for Scorpius X-1. 
We find that the predicted torque-balance ${ }^{11}$ limit could be reachable for the first time with an Einstein@Home search using data from a 6-month "Mid" aLIGO run (currently planned for 2016-2017), and should be beatable over a substantial frequency range in "Final" aLIGO. ${ }^{12}$ However, the frequency range over which the torque-balance limit can be beaten depends strongly on the Scorpius X-1 parameterspace uncertainty, most notably the orbital eccentricity and the assumed characteristics of spin wandering. As an example, by employing the Fast-Fourier-Transform resampling method ${ }^{13}$, assuming negligible uncertainty on $e$, we can beat the torquebalance level over a wide frequency range $[20,630] \mathrm{Hz}$ (for $\Delta T \leq 10 \mathrm{~d}$ ) or up to $\sim 500 \mathrm{~Hz}$ (for $\Delta T \leq 3 \mathrm{~d}$ ), and can still beat the torque-balance limit (albeit only up to a smaller frequency of $\sim 160 \mathrm{~Hz}-200 \mathrm{~Hz}$, depending on the constraint on $\Delta T$ ) in the case of substantial uncertainty ${ }^{14}$ on eccentricity $(\Delta e=0.087)$.

\section{Future Plans}

The results presented here build on and highlight the work of Ref. 9, and show the concrete possibility of beating the torque balance level for Scorpius X-1 by using the computing power of Einstein@Home for several months. We are currently undertaking a new challenge, consisting of developing a novel directed search strategy for CWs from binary systems, which exploits the peak-amplitude related statistic described in Ref. 15. This new algorithm is being conceived with the goal to overcome the hardy limitations dictated by computing resources. In a forthcoming paper we will validate the new method using software signal injections, and investigate its performance compared to current pipelines. ${ }^{4,9}$ With the advent of the advanced detector era it becomes essential to use different pipelines, which search for the same class of sources, and are implemented with independent software. This allows a robust vetting and accurate validation of results.

\section{References}

1. G. M. Harry, Classical Quantum Gravity 27, p. 084006 (2010).

2. V. Collab., https://tds.ego-gw.it/itf/tds/file.php?callFile=VIR-0027A-09.pdf .

3. A. Melatos and D. Payne, Astrophys.J. 623, 1044 (2005).

4. C. Messenger et al. arXiv:1504.05889, (2015).

5. P. Jaranowski et al., Phys.Rev. D58, p. 063001 (1998).

6. LIGO-Virgo Collaboration, Phys.Rev. D87, p. 042001 (2013).

7. R. Prix and M. Shaltev, 85, p. 084010 (2012).

8. R. Balasubramanian et al., Phys.Rev. D53, p. 3033 (1996).

9. P. Leaci and R. Prix, Phys.Rev. D91, p. 102003 (2015).

10. K. Wette, Phys.Rev. D85, p. 042003 (2012).

11. L. Bildsten, Astrophys. J. 501, p. L89 (1998).

12. D. S., https://dcc.ligo.org/cgi-bin/DocDB/ShowDocument?docid=T0900288 .

13. P. Patel et al., Phys.Rev. D81, p. 084032 (2010).

14. S. Premachandra. private communication, (2014).

15. P. Astone et al., Phys. Rev. D90, p. 042002 (2014). 